\section{Práticas contraceptivas e iniciação sexual entre jovens de três capitais brasileiras}

\author{
Contraceptive practices and sexual initiation \\ among young people in three Brazilian State \\ capitals
}

${ }_{1}^{1}$ Instituto de Saúde Coletiva, Universidade Federal da Bahia, Salvador, Brasil.

Correspondência L. F. B. Marinho MUSA - Programa Integrado de Pesquisa e Cooperação Técnica em Gênero e Saúde, Instituto de Saúde Coletiva Universidade Federal da Bahia.

Rua Basílio da Gama s/n, Campus Universitário do Canela, Salvador, BA 40110-040, Brasil. musa@ufba.br

\begin{abstract}
This study investigated contraceptive use during first sexual intercourse among 2.790 young men and women. The GRAVAD household survey in three Brazilian capital cities involved interviews in a probabilistic sample. A hierarchical logistic regression analysis was used. Variables grouped as: macro-social, socialization and sexual initiation, context of sexual initiation, and characteristics of the interviewee and his or her partner. The prevalence of contraceptive use was $68.3 \%$ for women and $65.3 \%$ for men. Among women, contraception use was associated to: per capita monthly family income, color/race, and the use of women's magazines as a source of information on pregnancy and contraception. For both genders, use was more frequent when partners discussed pregnancy prevention before intercourse, when sexual initiation was delayed and in a motel, and when the partner was patient. The interval between the start of the relationship and sexual initiation appeared associated to use during sexual initiation for men. Results suggest that macro-social factors determine more frequent contraception use during sexual initiation for women, while for men the relational context is more important.
\end{abstract}

Contraception Behavior; Sexuality; Gender Identity
Lilian F. B. Marinho 1

Estela M. L. Aquino 1

Maria da Conceição C. de Almeida ${ }^{1}$

\section{Introdução}

As práticas contraceptivas na iniciação sexual são diversificadas entre países, sobretudo naqueles em que se assegura o fornecimento dos insumos. Contudo, mesmo isso não exclui desigualdades entre grupos sociais e diferenças no interior destes 1 .

Nos países desenvolvidos e em desenvolvimento, a maioria dos jovens tem sua primeira relação sexual antes dos 20 anos, grande parcela antes dos 18, constatando-se historicamente a tendência à diminuição da idade e das diferenças entre as experiências de homens e mulheres 2,3,4,5. Entretanto, a idéia de "precocidade" da iniciação sexual é recorrente na literatura da demografia e da saúde coletiva, sendo apoiada por argumentos médicos relativos ao momento ideal para a primeira gravidez e à vulnerabilidade dos jovens às DST/AIDS 6,7.

Ao se investigar o uso de contracepção na primeira relação sexual é necessário considerar a multiplicidade de fatores envolvidos em uma prática que é sobretudo relacional ${ }^{8}$. Entretanto, freqüentemente os estudos demográficos têm se limitado a investigar macrodeterminantes, pela análise da relação entre renda per capita e nível de escolaridade com o uso de preservativos na iniciação sexual, e a descrever a prevalência de contracepção segundo indicadores clássicos, tais como faixa etária, conhecimento, fonte de informação e de obtenção dos métodos, bem como 
os tipos de contraceptivos utilizados e as razões para o não uso ${ }^{7}$. Sua realização sistemática tem sido fonte preciosa de monitoramento do uso de métodos, mas elucida pouco os fatores microssociais envolvidos na adoção de práticas contraceptivas.

$\mathrm{Na}$ área da saúde, até recentemente, os estudos concentravam-se em usuárias de serviços públicos de saúde, focalizando apenas mulheres, em geral adultas, e desconsiderando os papéis dos parceiros ou obtendo estas informações com base nas próprias mulheres. O pouco conhecimento sobre o papel masculino na negociação e/ou adoção de práticas preventivas na experimentação sexual e na reprodução convivia com mensagens veiculadas no campo da saúde pública sobre a responsabilidade mútua quanto à dupla proteção ou ao sexo seguro.

No Brasil, os primeiros inquéritos populacionais sobre comportamento sexual e reprodutivo, envolvendo jovens de ambos os sexos, foram realizados no final da década de 1980, constatando-se baixa prevalência (23\%) de uso de métodos contraceptivos na iniciação sexual entre as cidades pesquisadas 7 . Inquéritos mais recentes evidenciam um rápido e generalizado aumento do uso de contracepção na iniciação sexual, particularmente pelo uso de preservativo. Os resultados de dois inquéritos nacionais revelam o aumento da prevalência de uso do preservativo na iniciação sexual de 47,8\% em 1998 para 65,6\% em $2005^{3}$.

Desde os anos de 1990, estudos socioantropológicos têm investigado a sexualidade, contemplando elementos relacionais e contextuais quanto às práticas de prevenção da AIDS, com ênfase no uso do preservativo masculino ${ }^{8}$. Esses estudos abordam questões como a natureza dos relacionamentos, a diversidade de motivações para as práticas sexuais e de proteção, a avaliação subjetiva de riscos, e a complexidade do processo de decisão para a adoção de práticas preventivas.

Há evidências de que a adoção de determinado método ou mesmo o não uso deste visa a atender às preferências de pelo menos um dos parceiros sexuais 1,9 , o que pode ser potencializado em um momento crucial como o da iniciação sexual. De todo modo, mudanças e permanências precisam ser mais bem investigadas. Nessa direção, gênero é um demarcador para as diferenças encontradas na iniciação sexual que pode influenciar trajetórias afetivo-sexuais e o correspondente uso de contracepção 10 .

Este estudo tem por objetivo estimar o uso de contraceptivos na iniciação sexual de mulheres e homens jovens, além de identificar fatores associados à sua adoção, considerando-se desde os determinantes macrossociais até os microssociais, com especial contribuição relativamente às variáveis contextuais e relacionais. Entende-se que as práticas contraceptivas e de proteção são práticas sociais inscritas em relações hierárquicas e assimétricas de gênero, bem como de classe e raça/etnia, as quais são modeladas por normas sexuais e contraceptivas prescritas a partir de vários discursos: o da medicina, o da religião, o da demografia, o da educação, entre outros. Tratase, neste artigo, de empreender um esforço para fazer a travessia das explicações do contexto biomédico, cuja preocupação é o risco de gravidez na adolescência e de transmissão de HIV/AIDS e DST, para uma apreensão do fenômeno numa perspectiva que considere o contexto social mais amplo, e não apenas as questões restritas ao campo da sexualidade e da reprodução. Para isso, pretende-se integrar as contribuições dos estudos demográficos e sócio-antropológicos, o que exige uma estratégia que articule distintos níveis hierárquicos na análise.

\section{Material e métodos}

Trata-se de um estudo transversal, cujos dados são da Pesquisa GRAVAD - Gravidez na Adolescência: Estudo Multicêntrico sobre Jovens, Sexualidade e Reprodução no Brasil 11, realizada em Salvador (Bahia), Rio de Janeiro e Porto Alegre (Rio Grande do Sul).

A população-alvo é constituída de homens e mulheres de diferentes classes sociais, de 18 a 24 anos. O delineamento amostral compreendeu três estágios: seleção aleatória de no mínimo 55 setores censitários para cada cidade e de 33 domicílios por setor, com base na proporção de jovens em cada setor, no Censo Demográfico de 2000 (http://www.ibge.gov.br); e seleção, em cada domicílio sorteado, de uma pessoa dentro da faixa etária elegível. Os setores censitários foram agrupados em cinco estratos com base na renda média do chefe e proporção dos chefes com 12 ou mais anos de escolaridade. O parâmetro utilizado para o cálculo do tamanho da amostra foi a estimativa nacional da prevalência de no mínimo uma gravidez em mulheres de 18 e 19 anos, 25,3\% e $28,8 \%$, respectivamente 11 .

$\mathrm{O}$ inquérito envolveu 4.634 jovens dos sexos feminino e masculino de 18 a 24 anos, com taxa de resposta de $85,2 \%$. Neste estudo, foram considerados aqueles com idades de 20 a 24 anos, que tiveram a sua primeira relação sexual com pessoas do sexo oposto. A adoção de uma faixa etária subseqüente ao período definido como adolescência visou a permitir a reconstrução de trajetórias e a avaliação de eventuais escolhas 
reprodutivas, em um momento ainda próximo destes eventos.

Os dados foram produzidos entre outubro de 2001 e janeiro de 2002, por meio de entrevistas face a face aplicadas por entrevistadores de faixa etária próxima a dos entrevistados, com formação em Ciências Humanas.

Utilizou-se o Stata, versão 8.0 (Stata Corp., College Station, Estados Unidos) que permitiu incorporar o efeito do desenho amostral e os pesos relativos a cada unidade para a obtenção de estimativas confiáveis dos parâmetros.

Foi analisada a associação da variável dependente - uso de métodos na iniciação sexual - em relação a quatro níveis de variáveis (Figura 1), com base no aporte teórico da literatura sócioantropológica 8 , admitindo-se a existência de relações hierárquicas 12,13 entre elas quanto ao desfecho de interesse.

As diferenças entre os grupos foram avaliadas pelo teste de $\chi^{2}$ de Pearson, com a correção de segunda ordem de Rao e Scott. A seleção das variáveis para modelagem considerou um ní-

Figura 1

Modelo hierarquizado de análise do processo de determinação do uso de contraceptivos na primeira relação sexual, entre jovens de 20 a 24 anos.

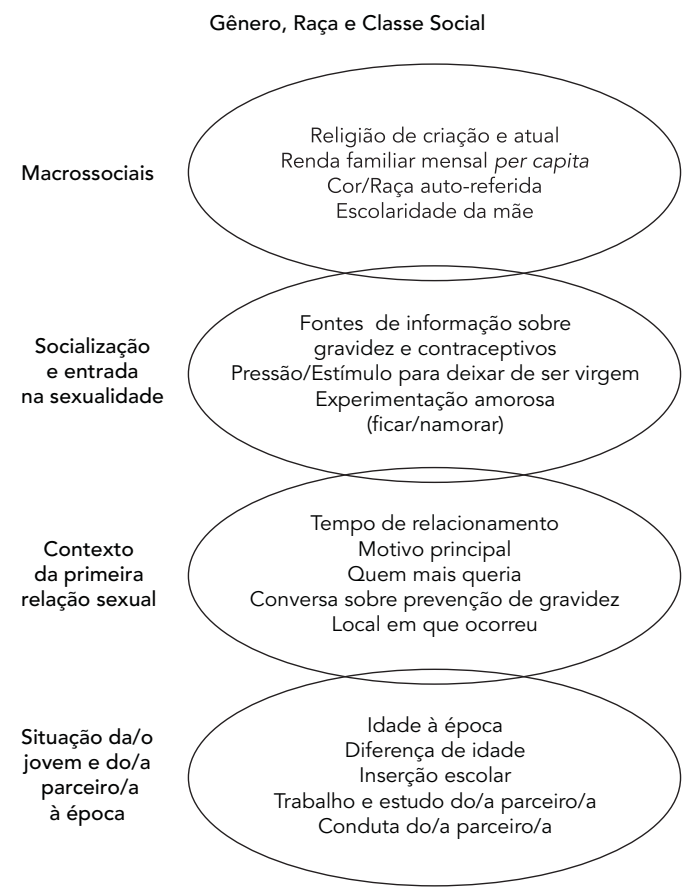

Práticas contraceptivas na iniciação sexual vel de significância estatística de até 0,20 . Na análise simultânea das variáveis de interesse, utilizou-se a odds ratio (OR) como medida de associação. A entrada no modelo considerou a OR bruta com significância menor que $10 \%$ e, em cada nível, permaneceram as variáveis que se mantiveram associadas ao uso de métodos, considerando a significância estatística no nível de $\mathrm{p}<0,05$ até a obtenção do modelo final. Para o cálculo das medidas ajustadas, utilizou-se a regressão logística multivariada, não condicional, em procedimento forward. A estratégia de entrada hierarquizada considerou inicialmente as variáveis do primeiro bloco, ajustando-as entre si, e as que permaneceram foram integradas às variáveis do segundo bloco. Tal procedimento foi repetido nas equações subseqüentes para a obtenção do modelo final.

A pesquisa foi cercada de cuidados éticos que envolveram a assinatura do termo de consentimento informado pelo/as entrevistado/as, bem como a garantia de privacidade e confidencialidade. O protocolo do estudo foi aprovado nos três centros de pesquisa envolvidos, pelos comitês de ética em pesquisa do Instituto de Saúde Coletiva, Universidade Federal da Bahia (ISC/UFBA); do Instituto de Medicina Social, Universidade do Estado do Rio de Janeiro (IMS/UERJ); e do Hospital de Clínicas de Porto Alegre,Universidade Federal do Rio Grande do Sul (UFRGS).

\section{Resultados}

Iniciação sexual: uma experiência marcada pelo gênero

Na Pesquisa GRAVAD 11, dos 3.029 jovens que tinham à época entre 20 e 24 anos, 88,7\% das mulheres e $93 \%$ dos homens referiram ter vivenciado sua primeira relação sexual com pessoas do sexo oposto, totalizando 2.790 jovens que constituíram a população do presente estudo.

A distribuição dos jovens segundo renda familiar per capita mostra que $39,2 \%$ das mulheres e $32 \%$ dos homens encontravam-se na faixa de até um salário mínimo. As moças tinham mais alta escolaridade: $58,5 \%$ delas e $49,5 \%$ deles tinham alcançado nível médio ou superior (mesmo incompleto). A grande maioria dos jovens foi criada no catolicismo $(75,1 \%$ das mulheres e $69,7 \%$ dos homens). Quase metade dos entrevistados declarou-se de cor preta ou parda, mas isto variou de $23 \%$ em Porto Alegre a $41,6 \%$ no Rio de Janeiro e $71,5 \%$ em Salvador.

Foi elevada a proporção de jovens que tiveram a experiência de "namorar e ficar" - 77,4\% entre as mulheres e $93,1 \%$ entre os homens. 
As moças tenderam a ter sua iniciação sexual em relações com maior estabilidade, com $77,2 \%$ aguardando no mínimo três meses para que isto acontecesse, enquanto $35,3 \%$ deles encontravam-se na mesma situação. Elas também tenderam a iniciar-se um pouco mais tarde: para $44,6 \%$ isso ocorreu aos 18 anos e mais, com idade mediana de 17,9 anos; para os rapazes esta foi de 16,2 anos e apenas 1/5 deles teve a primeira relação sexual aos 18 anos e mais. Mais da metade deles declarou ter tido sua iniciação sexual em um relacionamento eventual, mas o recurso a profissionais do sexo foi quase desprezível (4,2\%).

À época da entrevista, somente 33,8\% das mulheres referiram estar casadas ou unidas, $45 \%$ das quais com o mesmo parceiro da iniciação sexual; entre os homens estes valores foram de $20,7 \%$ e $10,4 \%$, respectivamente.

\section{Proteção para gravidez na primeira relação sexual}

A prevalência de contracepção estimada com base no relato das mulheres foi maior em Porto Alegre $(77,5 \%)$ e menor em Salvador $(60,6 \%)$ (Tabela 1). O preservativo masculino foi mais referido por elas e eles, sendo os maiores percentuais observados em Salvador, respectivamente, $89 \%$ e $81 \%$. A pílula ocupou a segunda posição alcançando o maior percentual entre as mulheres do Rio de Janeiro (19,9\%) e o menor em Porto Alegre $(12,6 \%)$ (dados não apresentados). Esse padrão é mantido para os rapazes, porém, com valores reduzidos à metade.

Em ambos os sexos, a prevalência do uso de métodos de contracepção aumentou com a renda familiar per capita. Foi mais elevada entre os brancos (mulheres: 76,5\%; homens: $72,2 \%$ ), sendo o menor valor observado entre as mulheres indígenas $(51,6 \%)$ e entre homens pretos/pardos $(58,7 \%)$. Os percentuais mais elevados foram das mulheres criadas no catolicismo $(71,8 \%)$ comparativamente às que mencionaram outro pertencimento religioso. Os homens apresentaram padrão semelhante com níveis limítrofes de significância estatística (Tabela 1). Maior prevalência de uso foi observada entre as moças $(52,1 \%)$ e rapazes $(57,1 \%)$ cujas mães tinham nível médio ou superior, ainda que incompleto.

A análise de fontes das primeiras informações sobre gravidez e meios para evitar filhos apresentou resultados curiosos. Ainda que a mãe tenha sido a mais citada, a associação com o uso da contracepção na primeira relação sexual não alcançou significância estatística entre mulheres, e manteve-se em nível limítrofe entre os homens. Ter a escola e os professores como informantes associou-se ao comportamento das moças, mas não ao dos rapazes. Ter o pai como fonte aumentou o uso de proteção pelos rapazes, o que foi também observado entre as mulheres em nível limítrofe de significância. Para elas, a prevalência do uso de métodos de contracepção foi maior $(83,5 \%)$ entre as que citaram revistas femininas como fontes das primeiras informações sobre meios para evitar gravidez (Tabela 1). É importante destacar que os médicos e os serviços de saúde não foram citados de modo expressivo como fontes primárias de informação sobre sexualidade e reprodução e, por isto, foram excluídos da análise pela falta de significância estatística.

A inexperiência do parceiro das mulheres não influenciou o uso da proteção na iniciação sexual, mas a prevalência de uso obtida a partir dos relatos masculinos reduziu-se a 40,6\% quando a parceira também era virgem. Porém, ter tido a iniciação sexual em relacionamento estável associou-se à elevada proteção para as mulheres (68,7\%), quando comparada com a ocorrência deste evento em relacionamento ocasional; entre os homens, menos da metade dos que se iniciaram em um relacionamento estável usou alguma proteção. A diferença de idade entre os parceiros mostrou-se associada com o relato do uso de contraceptivos somente entre as moças, com proporção mais elevada (72,7\%) quando a entrevistada tinha até 19 anos e o parceiro já ultrapassara esta idade, em comparação à situação em que ambos eram adolescentes $(61,8 \%)$ (Tabela 1$)$ Entre as mulheres e homens que declararam ter usado algum método contraceptivo, a freqüência foi maior quando a preocupação foi de ambos, respectivamente $69,6 \%$ e $73,9 \%$, do que de apenas um deles (dados não apresentados).

\section{A contracepção na iniciação sexual na perspectiva das mulheres}

A estratégia hierarquizada para análise simultânea dos fatores associados ao uso de contracepção na iniciação sexual iniciou-se no nível mais distal dos determinantes macrossociais. Nesse primeiro nível, após o ajuste, mantiveram-se associadas renda familiar per capita e cor/raça autodeclarada. Para quem informou renda acima de três salários mínimos, o uso de métodos contraceptivos foi duas vezes maior do que sendo a renda inferior a este limite. Ser de cor branca representou um acréscimo de $70 \%$ na proporção de uso, comparativamente às que referiram ser pretas/pardas e indígenas (Tabela 2).

No segundo nível, mantidas as variáveis renda familiar per capita e cor/raça auto-referida, foram incluídas aquelas relativas ao modo de socialização e entrada na sexualidade, estas últi- 
Prevalência do uso de métodos contraceptivos (P) na iniciação sexual entre jovens de 20 a 24 anos, segundo características selecionadas por sexo.

\begin{tabular}{|c|c|c|c|c|c|c|c|c|}
\hline \multirow[t]{2}{*}{ Características } & \multicolumn{4}{|c|}{ Mulheres } & \multicolumn{4}{|c|}{ Homens } \\
\hline & $n$ * & $\mathrm{P}(\%)$ & IC95\% & Valor de $p$ & $n$ * & $P(\%)$ & IC95\% & Valor de $p$ \\
\hline Prevalência bruta & 1.422 & 68,3 & $64,5-71,9$ & & 1.344 & 65,3 & $60,5-69,8$ & \\
\hline \multicolumn{9}{|l|}{ Cidade } \\
\hline Porto Alegre & 446 & 77,5 & $71,7-82,3$ & 0,0024 & 444 & 70,6 & $64,3-76,1$ & 0,2549 \\
\hline Rio de Janeiro & 451 & 70,1 & $64,3-75,4$ & & 457 & 65,9 & $58,3-72,8$ & \\
\hline Salvador & 525 & 60,6 & $54,7-66,1$ & & 443 & 61,5 & $56,0-66,6$ & \\
\hline \multicolumn{9}{|l|}{ Renda familiar mensal per capita ** } \\
\hline Até $\mathrm{R} \$ 90,00$ & 234 & 58,7 & $47,6-69,0$ & 0,0000 & 141 & 53,6 & $42,0-64,8$ & 0,0783 \\
\hline $\mathrm{R} \$ 91,00$ a $\mathrm{R} \$ 180,00$ & 282 & 54,8 & $46,2-63,1$ & & 227 & 66,8 & $56,9-75,5$ & \\
\hline $\mathrm{R} \$ 181,00$ a $\mathrm{R} \$ 540,00$ & 463 & 70,6 & $65,5-75,2$ & & 521 & 65,0 & $57,6-71,7$ & \\
\hline Acima de $\mathrm{R} \$ 540,00$ & 437 & 83,3 & $78,2-87,4$ & & 455 & 70,4 & $64,3-75,8$ & \\
\hline \multicolumn{9}{|l|}{ Religião em que foi criada/o } \\
\hline Católica & 1.071 & 71,8 & $67,4-75,8$ & 0,0024 & 946 & 69,1 & $63,7-74,0$ & 0,0688 \\
\hline Pentecostal, protestante & 93 & 56,1 & $41,0-70,1$ & & 92 & 57,9 & $43,9-70,7$ & \\
\hline $\begin{array}{l}\text { Espírita, umbanda, candomblé, } \\
\text { batuque, outra }\end{array}$ & 160 & 63,5 & $53,6-72,3$ & & 158 & 56,2 & $41,6-69,7$ & \\
\hline Nenhuma & 93 & 48,9 & $34,4-63,3$ & & 141 & 58,5 & $48,5-68,0$ & \\
\hline \multicolumn{9}{|l|}{ 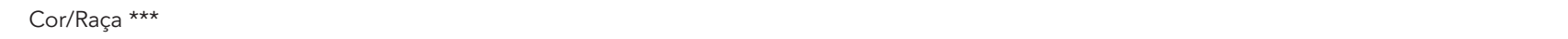 } \\
\hline Branca & 701 & 76,5 & $71,6-80,9$ & & 693 & 72,2 & $60,5-69,9$ & \\
\hline Preta/parda & 612 & 62,2 & $57,2-67,0$ & 0,0012 & 549 & 58,7 & $52,4-64,7$ & 0,0026 \\
\hline Indígena & 73 & 54,6 & $35,2-72,6$ & & 85 & 68,3 & $55,9-78,6$ & \\
\hline \multicolumn{9}{|l|}{ Escolaridade da mãe } \\
\hline $\begin{array}{l}\text { Até Ensino Fundamental } \\
\text { completo }\end{array}$ & 582 & 47,9 & $42,1-53,8$ & 0,0149 & 457 & 42,9 & $36,2-49,9$ & 0,0055 \\
\hline $\begin{array}{l}\text { Médio ou superior } \\
\text { (mesmo incompleto) }\end{array}$ & 610 & 52,1 & $46,2-57,9$ & & 671 & 57,1 & $50,1-63,7$ & \\
\hline \multicolumn{9}{|l|}{ Fontes de informações sobre } \\
\hline \multicolumn{9}{|l|}{ gravidez e meios para evitar } \\
\hline Mãe & 619 & 72,0 & $66,5-77,0$ & 0,1284 & 522 & 71,9 & $62,2-69,9$ & 0,0516 \\
\hline Pai & 115 & 79,6 & $67,6-87,9$ & 0,0550 & 366 & 73,8 & $64,9-81,1$ & 0,0086 \\
\hline Professor/Escola & 463 & 74,9 & $69,2-79,8$ & 0,0065 & 435 & 62,9 & $56,7-68,7$ & 0,3719 \\
\hline Revistas femininas & 172 & 83,5 & $75,2-89,9$ & 0,0006 & 41 & 56,1 & $32,7-77,1$ & 0,3978 \\
\hline \multicolumn{9}{|l|}{ Primeira vez do/a parceiro/a } \\
\hline Sim & 186 & 72,9 & $63,2-80,8$ & 0,3044 & 479 & 66,1 & $59,3-72,3$ & 0,4305 \\
\hline Não & 1.200 & 68,0 & $63,8-71,9$ & & 721 & 63,7 & $58,1-68,9$ & \\
\hline \multicolumn{9}{|l|}{ Natureza do relacionamento } \\
\hline Estável & 1.277 & 68,7 & $64,9-72,3$ & & 727 & 62,9 & $56,8-68,7$ & \\
\hline Ocasional & 143 & 64,6 & $53,2-74,5$ & 0,4150 & 609 & 68,1 & $62,3-73,3$ & 0,1509 \\
\hline \multicolumn{9}{|l|}{ Intervalo de tempo entre o início do } \\
\hline \multicolumn{9}{|c|}{ relacionamento e a iniciação sexual (dias) } \\
\hline$<90$ & 332 & 67,3 & $58,8-74,9$ & 0,6908 & 829 & 59,3 & $53,6-64,8$ & 0,0001 \\
\hline $90-364$ & 635 & 70,6 & $64,8-75,8$ & & 346 & 76,2 & $69,2-82,0$ & \\
\hline 365 e mais & 430 & 67,4 & $60,9-72,5$ & & 75 & 64,6 & $49,4-76,5$ & \\
\hline \multicolumn{9}{|l|}{ Diferença de idade entre os parceiros } \\
\hline Ambos adolescentes & 550 & 61,8 & $55,7-67,6$ & 0,0034 & 994 & 64,0 & $58,3-69,4$ & 0,2670 \\
\hline $\begin{array}{l}\text { Adolescente com parceiro/a } \\
\text { acima de } 20 \text { anos }\end{array}$ & 848 & 72,7 & $67,9-76,9$ & & 246 & 68,7 & $60,7-75,7$ & \\
\hline
\end{tabular}

População: jovens de 20 a 24 anos, Porto Alegre (Rio Grande do Sul), Rio de Janeiro, Salvador (Bahia).

* A soma dos totais difere devido à perda de informações em algumas variáveis;

** Valor do salário mínimo à época do inquérito = R \$180,00;

*** Exclusão de 47 entrevistadas/os que se declararam de cor/raça amarela. 
mas posteriormente excluídas do modelo após o ajuste, à exceção da menção a revistas femininas como fonte de informação sobre gravidez e contraceptivos (Tabela 2).

No terceiro nível concernente ao contexto da iniciação sexual e do uso de contraceptivos, a conversa com o parceiro sobre prevenção de gravidez antes da iniciação sexual e o lugar em que esta ocorreu mantiveram-se associados após o ajuste para as demais variáveis.

Por fim, entre as variáveis do nível mais proximal, apenas a idade à época da iniciação sexual e a conduta do parceiro no momento permaneceram associadas ao uso de proteção (Tabela 2).

Vale ressaltar que, conforme os critérios estatísticos adotados, algumas variáveis, apesar de importantes na literatura, não se mantiveram no modelo, a exemplo de religião de criação, diferença de idade entre os parceiros, natureza da relação e tempo de relacionamento.

\section{A perspectiva dos homens sobre a proteção na iniciação sexual}

Diferentemente do observado entre as mulheres, no nível sociofamiliar, a renda familiar per capita não teve influência sobre o uso de mé- todos contraceptivos na iniciação sexual, entre os homens, e apenas a cor/raça auto-referida mostrou-se associada (Tabela 3).

No segundo nível, relativo ao modo de socialização e entrada na sexualidade, nenhuma variável teve influência quando procedido o ajuste pelas demais. Entretanto, mostraram-se importantes para a proteção na iniciação sexual as variáveis do contexto relacional - o tempo do relacionamento, o local em que ocorreu e a conversa prévia com a parceira sobre o assunto, esta última sendo a mais relevante. Também a idade do jovem à época e a conduta da parceira mantiveram-se associadas à proteção. Assim, ter tido a primeira relação sexual aos três meses ou mais depois de iniciado o relacionamento afetivo resultou em incremento de $80 \%$ relativamente ao menor tempo; ter conversado com a parceira sobre prevenção de gravidez mostrou-se quase cinco vezes mais associado à declaração do uso de métodos contraceptivos. Também teve influência positiva o fato da iniciação sexual ter ocorrido em motel, em contraste com os demais locais (Tabela 3).

Como observado entre as mulheres, a idade à época da primeira relação sexual mostrouse fortemente associada ao uso de métodos

Análise hierarquizada por regressão logística dos fatores associados ao uso de métodos contraceptivos entre mulheres na primeira relação sexual.

\begin{tabular}{|c|c|c|c|c|c|c|c|}
\hline \multirow[t]{3}{*}{ Variáveis } & \multirow[t]{3}{*}{$n$ * } & \multirow{3}{*}{$\begin{array}{l}\text { Uso de } \\
\text { métodos } \\
\text { (\%) }\end{array}$} & \multicolumn{5}{|c|}{ OR (IC95\%) } \\
\hline & & & \multirow[t]{2}{*}{ Bruta } & \multicolumn{4}{|c|}{ Ajustada } \\
\hline & & & & Nível I ** & Nível II *** & Nível III \# & Nível IV \#\# \\
\hline \multicolumn{8}{|l|}{ Macrossocial } \\
\hline \multicolumn{8}{|l|}{ Renda familiar mensal per capita \#\#\# } \\
\hline Até $\mathrm{R} \$ 540,00$ & 985 & 63,2 & 1,0 & 1,0 & 1,0 & 1,0 & 1,0 \\
\hline $\mathrm{R} \$ 541,00$ e mais & 437 & 83,3 & $2,9(2,0-4,3)$ & $2,0(1,3-3,1)$ & $2,0(1,4-3,0)$ & $2,0(1,4-3,0)$ & $1,8(1,2-2,8)$ \\
\hline \multicolumn{8}{|l|}{ Escolaridade da mãe } \\
\hline Até ensino fundamental completo & 813 & 64,8 & 1,0 & 1,0 & & & \\
\hline Médio ou superior (mesmo incompleto) & 544 & 77,1 & $1,8(1,2-2,7)$ & $1,2(0,8-1,8)$ & & & \\
\hline \multicolumn{8}{|l|}{ Criação em alguma religião } \\
\hline Não & 93 & 48,8 & 1,0 & 1,0 & & & \\
\hline Sim & 1324 & 69,5 & $2,4(1,3-4,5)$ & $1,7(0,9-3,4)$ & & & \\
\hline \multicolumn{8}{|l|}{ Cor/Raça (auto-referida) § } \\
\hline Preta, parda, indígena & 685 & 61,2 & 1,0 & 1,0 & 1,0 & 1,0 & 1,0 \\
\hline Branca & 701 & 76,6 & $2,1(1,5-2,9)$ & $1,7(1,2-2,4)$ & $1,6(1,0-2,4)$ & $1,9(1,3-2,7)$ & $1,9(1,3-2,7)$ \\
\hline \multicolumn{8}{|l|}{ Socialização e entrada na sexualidade } \\
\hline \multicolumn{8}{|l|}{ Obteve informações sobre gravidez } \\
\hline \multicolumn{8}{|l|}{ e métodos contraceptivos com } \\
\hline \multicolumn{8}{|l|}{ Mãe } \\
\hline $\operatorname{Sim}$ & 619 & 72,0 & $1,3(0,9-1,9)$ & & $1,2(0,8-1,7)$ & & \\
\hline Não & 801 & 65,9 & 1,0 & & 1,0 & & \\
\hline
\end{tabular}

(continua) 
Tabela 2 (continuação)

\begin{tabular}{|c|c|c|c|c|c|c|c|}
\hline \multirow[t]{3}{*}{ Variáveis } & \multirow[t]{3}{*}{$n$ * } & \multirow{3}{*}{$\begin{array}{l}\text { Uso de } \\
\text { métodos } \\
\text { (\%) }\end{array}$} & \multicolumn{5}{|c|}{ OR (IC95\%) } \\
\hline & & & \multirow[t]{2}{*}{ Bruta } & \multicolumn{4}{|c|}{ Ajustada } \\
\hline & & & & Nível I ** & Nível II *** & Nível III \# & Nível IV \#\# \\
\hline \multicolumn{8}{|l|}{ Pai } \\
\hline Sim & 115 & 79,6 & $1,9(1,0-3,6)$ & & 1,0 & & \\
\hline Não & 1.305 & 67,5 & 1,0 & & $1,2(0,6-2,3)$ & & \\
\hline \multicolumn{8}{|l|}{ Professor/Escola } \\
\hline Sim & 463 & 74,9 & $1,5(1,1-2,1)$ & & $1,5(1,0-2,1)$ & $1,2(0,9-1,9)$ & \\
\hline Não & 957 & 65,8 & 1,0 & & 1,0 & 1,0 & \\
\hline \multicolumn{8}{|l|}{ Revistas femininas } \\
\hline Sim & 172 & 83,8 & $2,6(1,5-4,5)$ & & $2,1(1,2-3,8)$ & $2,6(1,3-4,9)$ & $2,2(1,1-4,1)$ \\
\hline Não & 1.248 & 66,5 & 1,0 & & 1,0 & 1,0 & 1,0 \\
\hline \multicolumn{8}{|c|}{ Pressão/Estímulo para deixar } \\
\hline \multicolumn{8}{|l|}{ de ser virgem } \\
\hline Não & 1.113 & 70,5 & $1,7(1,2-2,5)$ & & $1,8(1,3-2,6)$ & $1,3(0,9-2,0)$ & \\
\hline Sim & 287 & 58,0 & 1,0 & & 1,0 & 1,0 & \\
\hline \multicolumn{8}{|c|}{ Contexto da iniciação sexual } \\
\hline \multicolumn{8}{|c|}{ Quem mais queria a iniciação sexual } \\
\hline Os dois igualmente & 999 & 71,7 & $1,7(1,2-2,3)$ & & & $1,5(1,0-2,1)$ & \\
\hline Ela ou o parceiro & 414 & 59,9 & 1,0 & & & 1,0 & \\
\hline \multicolumn{8}{|c|}{ Conversa com o parceiro sobre } \\
\hline \multicolumn{8}{|l|}{ prevenção de gravidez } \\
\hline Sim & 851 & 82,6 & $5,6(3,9-8,1)$ & & & $5,3(3,7-7,6)$ & $4,5(3,0-6,5)$ \\
\hline Não & 567 & 45,8 & 1,0 & & & 1,0 & \\
\hline \multicolumn{8}{|l|}{ Lugar em que ocorreu } \\
\hline Motel/Hotel & 268 & 85,2 & $3,3(2,0-5,3)$ & & & $2,8(1,6-4,9)$ & $2,5(1,5-4,4)$ \\
\hline Outros $\S \S$ & 1.140 & 63,8 & 1,0 & & & 1,0 & \\
\hline \multicolumn{8}{|c|}{ Motivo para que ocorresse } \\
\hline Amor & 717 & 74,2 & $1,8(1,3-2,4)$ & & & $0,9(0,6-1,5)$ & \\
\hline Outros §£§ & 686 & 62,0 & 1,0 & & & 1,0 & \\
\hline \multicolumn{8}{|c|}{ Situação da jovem e do parceiro à } \\
\hline \multicolumn{8}{|c|}{ época da iniciação sexual } \\
\hline \multicolumn{8}{|l|}{ Idade da jovem (anos) } \\
\hline 15 e menos & 374 & 46,9 & 1,0 & & & & 1,0 \\
\hline 16 e 17 & 456 & 70,7 & $2,7(1,8-4,1)$ & & & & $1,7(1,1-2,7)$ \\
\hline 18 e mais & 586 & 79,1 & $4,3(2,8-6,6)$ & & & & $2,0(1,2-3,3)$ \\
\hline \multicolumn{8}{|l|}{ Parceiro estudava } \\
\hline Não & 679 & 65,0 & 1,0 & & & & \\
\hline Sim & 734 & 71,9 & $1,4(1,0-1,9)$ & & & & \\
\hline \multicolumn{8}{|l|}{ Conduta do parceiro $\neq$} \\
\hline Impaciente & 195 & 48,8 & 1,0 & & & & 1,0 \\
\hline Paciente & 1.214 & 71,5 & $2,6(1,6-4,4)$ & & & & $1,6(1,0-2,7)$ \\
\hline
\end{tabular}

Fonte: Pesquisa GRAVAD, 2002.

População: jovens de 20 a 24 anos, Porto Alegre (Rio Grande do Sul), Rio de Janeiro, Salvador (Bahia).

* A soma dos totais difere devido à perda de informações em algumas variáveis;

** Ajustado incluindo variáveis do nível 1 (sócio-familiares);

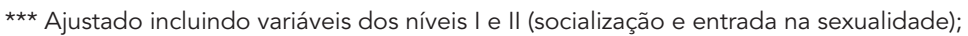

\# Ajustado para variáveis dos níveis I, II e III (contexto da primeira relação sexual e uso de contraceptivos);

\#\# Ajustado para variáveis dos níveis I, II, III e IV (situação da jovem e do parceiro à época);

\#\#\# Valor do salário mínimo à época do inquérito = R\$180,00;

$\S$ Exclusão de 20 entrevistadas que se declararam de cor/raça amarela;

$\S \S$ Inclui: própria casa, do parceiro, de amigos/conhecidos, festa/baile, carro, praia, rua/lugares públicos, outros;

$\S \S \S$ Inclui: curiosidade, tesão, medo de perder o companheiro, vontade de perder logo a virgindade e outros;

‡ Paciente: inclui atencioso, tranqüilo, calmo; impaciente: inclui apressado, nervoso, assustado. 
Análise hierarquizada por regressão logística dos fatores associados ao uso de métodos contraceptivos entre homens na primeira relação sexual.

\begin{tabular}{|c|c|c|c|c|c|c|c|}
\hline \multirow[t]{3}{*}{ Variáveis } & \multirow[t]{3}{*}{$n$ * } & \multirow{3}{*}{$\begin{array}{l}\text { Uso de } \\
\text { métodos } \\
\text { (\%) }\end{array}$} & \multicolumn{5}{|c|}{ OR (IC95\%) } \\
\hline & & & \multirow[t]{2}{*}{ Bruta } & \multicolumn{4}{|c|}{ Ajustada } \\
\hline & & & & Nível I ** & Nível II *** & Nível III \# & Nível IV \#\# \\
\hline \multicolumn{8}{|l|}{ Macrossocial } \\
\hline \multicolumn{8}{|l|}{ Renda familiar mensal per capita \#\#\# } \\
\hline Até $\mathrm{R} \$ 540,00$ & 889 & 63,4 & $1,4(0,9-2,0)$ & $1,1(0,7-1,6)$ & & & \\
\hline $\mathrm{R} \$ 541,00$ e mais & 455 & 70,4 & 1,0 & 1,0 & & & \\
\hline \multicolumn{8}{|l|}{ Escolaridade da mãe } \\
\hline Até ensino fundamental completo & 673 & 62,7 & 1,0 & 1,0 & & & \\
\hline Médio ou superior (mesmo incompleto) & 586 & 70,1 & $1,4(1,0-2,0)$ & $1,1(0,8-1,6)$ & & & \\
\hline \multicolumn{8}{|l|}{ Criação em alguma religião } \\
\hline Sim & 1.196 & 66,5 & $1,4(0,8-2,4)$ & $1,5(0,9-2,6)$ & & & \\
\hline Não & 141 & 58,5 & 1,0 & & & & \\
\hline \multicolumn{8}{|l|}{ Cor/Raça (autoclassificação) § } \\
\hline Branca & 693 & 72,2 & $1,7(1,1-2,5)$ & $1,6(1,1-2,5)$ & $1,6(1,1-2,5)$ & $1,6(1,0-2,4)$ & $1,5(1,0-2,2)$ \\
\hline Preta, parda, indígena & 634 & 59,9 & 1,0 & 1,0 & 1,0 & 1,0 & 1,0 \\
\hline \multicolumn{8}{|l|}{ Socialização e entrada na sexualidade } \\
\hline \multicolumn{8}{|l|}{ Obteve informações sobre gravidez } \\
\hline \multicolumn{8}{|l|}{ e métodos contraceptivos com } \\
\hline \multicolumn{8}{|l|}{ Mãe } \\
\hline Sim & 522 & 71,9 & $1,6(1,0-2,6)$ & & $1,4(0,8-2,3)$ & & \\
\hline Não & 814 & 61,4 & 1,0 & & 1,0 & & \\
\hline \multicolumn{8}{|l|}{ Pai } \\
\hline Sim & 366 & 73,8 & $1,7(1,2-2,5)$ & & $1,3(0,9-1,9)$ & & \\
\hline Não & 970 & 62,5 & 1,0 & & 1,0 & & \\
\hline \multicolumn{8}{|l|}{ Contexto da iniciação sexual } \\
\hline \multicolumn{8}{|l|}{ Quem mais queria a iniciação sexual } \\
\hline Ele ou a parceira & 325 & 59,2 & 1,0 & & & & \\
\hline Os dois igualmente & 943 & 65,8 & $1,3(0,9-1,9)$ & & & & \\
\hline \multicolumn{8}{|l|}{ Tempo decorrido entre o início do } \\
\hline relacionamento e a iniciação sexual & & & & & & & \\
\hline Menos de três meses & 829 & 59,3 & 1,0 & & & 1,0 & 1,0 \\
\hline Três meses ou mais & 421 & 74,1 & $2,0(1,4-2,8)$ & & & $1,8(1,1-3,0)$ & $1,7(1,1-2,6)$ \\
\hline Conversa com a parceira sobre & & & & & & & \\
\hline prevenção de gravidez & & & & & & & \\
\hline Não & 825 & 53,5 & 1,0 & & & 1,0 & 1,0 \\
\hline Sim & 514 & 84,3 & $4,7(3,3-6,7)$ & & & $4,9(3,2-7,5)$ & $4,4(2,9-6,7)$ \\
\hline Lugar em que ocorreu & & & & & & & \\
\hline Outros $\S \S$ & 1.196 & 61,5 & 1,0 & & & 1,0 & 1,0 \\
\hline Motel/hotel & 92 & 89,7 & $5,4(2,5-11,7)$ & & & $4,9(2,2-10,9)$ & $3,7(1,5-9,5)$ \\
\hline Motivo para que ocorresse & & & & & & & \\
\hline Outros $\S \S \S$ & 1.174 & 63,2 & 1,0 & & & 1,0 & \\
\hline Amor & 156 & 78,6 & $2,1(1,3-3,4)$ & & & $1,2(0,6-2,2)$ & \\
\hline Natureza da relação ¥ & & & & & & & \\
\hline Eventual & 727 & 62,9 & 1,0 & & & & \\
\hline Estável & 609 & 68,0 & $1,2(0,9-1,7)$ & & & & \\
\hline Situação do jovem e da parceira à & & & & & & & \\
\hline época da iniciação sexual & & & & & & & \\
\hline Idade do jovem (anos) & & & & & & & \\
\hline$\leq 14$ & 380 & 45,8 & 1,0 & & & & 1,0 \\
\hline $15-16$ & 523 & 72,1 & $3,1(2,0-4,6)$ & & & & $3,1(2,0-4,8)$ \\
\hline 17 e mais & 434 & 73,8 & $3,3(2,1-5,2)$ & & & & $2,4(1,5-4,0)$ \\
\hline
\end{tabular}

(continua) 


\begin{tabular}{|c|c|c|c|c|c|c|c|}
\hline \multirow[t]{3}{*}{ Variáveis } & \multirow[t]{3}{*}{$n$ * } & \multirow{3}{*}{$\begin{array}{l}\text { Uso de } \\
\text { métodos } \\
(\%)\end{array}$} & \multicolumn{5}{|c|}{ OR (IC95\%) } \\
\hline & & & \multirow[t]{2}{*}{ Bruta } & \multicolumn{4}{|c|}{ Ajustada } \\
\hline & & & & Nível I ** & Nível II *** & Nível III \# & Nível IV \#\# \\
\hline \multicolumn{8}{|c|}{ Parceira trabalhava } \\
\hline Não & 914 & 62,2 & 1,0 & & & & \\
\hline Sim & 282 & 68,2 & $1,3(0,9-1,9)$ & & & & \\
\hline \multicolumn{8}{|c|}{ Conduta da parceira 㧊 } \\
\hline Impaciente & 898 & 57,5 & 1,0 & & & & 1,0 \\
\hline Paciente & 437 & 69,6 & $1,7(1,2-2,4)$ & & & & $2,2(1,4-3,3$ \\
\hline
\end{tabular}

Fonte: Pesquisa GRAVAD, 2002.

População: Jovens de 20 a 24 anos, Porto Alegre (Rio Grande do Sul), Rio de Janeiro, Salvador (Bahia).

* A soma dos totais difere devido à perda de informações em algumas variáveis;

** Ajustado incluindo variáveis do nível 1 (sócio-familiares);

*** Ajustado incluindo variáveis dos níveis I e II (socialização e entrada na sexualidade);

\# Ajustado para variáveis dos níveis I, II e III (contexto da primeira relação sexual e uso de contraceptivos);

\#\# Ajustado para variáveis dos níveis I, II, III e IV (situação do jovem e da parceira à época);

\#\# Valor do salário mínimo à época do inquérito $=\mathrm{R} \$ 180,00$;

$\S$ Exclusão de 9 entrevistados que se declararam de cor/raça amarela;

$\S \S$ Inclui: própria casa, do parceiro, de amigos/conhecidos, festa/baile, carro, praia, rua/lugares públicos, outros;

$\S \S \S$ Inclui: curiosidade, tesão, medo de perder a companheira, vontade de perder logo a virgindade e outros;

‡ Estável: namorada, esposa ou companheira; ocasional: pessoa com quem você ficou, garota de programa/ profissional do sexo ou outros

¥ Paciente inclui: atenciosa, tranqüila, calma; impaciente inclui: apressada, nervosa, assustada.

contraceptivos, assim como uma conduta paciente, tranqüila, calma e atenciosa da parceira (Tabela 3).

\section{Discussão}

A inclusão de homens constituiu um aspecto relevante do presente estudo, permitindo abordar questões relacionais, tanto na perspectiva masculina quanto na feminina.

Os limites dos estudos transversais encontram-se bem documentados na literatura epidemiológica, a exemplo da possibilidade da existência de viés de memória. Todavia, alguns aspectos desta investigação podem ter contribuído para relativizá-lo, merecendo destaque: além de tratar-se de uma população jovem, o intervalo de tempo decorrido entre os eventos de interesse e o momento da entrevista foi relativamente curto. A estratégia de buscar a reconstrução das trajetórias afetivo-sexuais também se constitui num ponto forte, favorecendo a rememoração dos eventos.

Há que se considerar que a iniciação sexual e as práticas contraceptivas são eventos investigados necessariamente por meio do relato dos envolvidos, que podem ter sua importância e significados reinterpretados por eles/elas 14, influenciando a produção de respostas socialmen- te aceitáveis. Uma eventual superestimação da prevalência do uso de contraceptivos e/ou proteção na iniciação sexual não pode ser completamente afastada, em virtude da epidemia da AIDS e a ampla divulgação da importância do uso de preservativos em todas as relações sexuais, como "sexo seguro". Mas, os achados são coerentes com estudos recentes, que indicam tendência crescente do uso de métodos na iniciação sexual, embora com diminuição do uso na medida em que os relacionamentos se estabelecem 3,15 .

Um número expressivo de jovens teve sua primeira relação sexual antes dos 20 anos e permaneciam solteiros à época da entrevista. A exemplo do que se observa em outros achados 3 , a iniciação sexual parece não se tratar de uma iniciativa individual e autônoma: os rapazes sentiram-se mais pressionados do que as moças a deixarem de ser virgens, sendo maior a pressão externa ao casal, atribuída aos amigos, enquanto as moças apontaram os parceiros como aqueles que mais as pressionaram. O relato delas pode refletir a ambivalência entre o desejo e a norma social ainda vigente em alguns contextos sociais, que considera a iniciativa como prerrogativa masculina, devendo as mulheres resistir, para posteriormente ceder 14. De todo modo, é necessário investigar as estratégias de acomodação e resistência aos estereótipos dos papéis sexuais desempenhados por mulheres e homens. 
O "ficar" como um marco organizador na entrada da sexualidade mostrou-se mais freqüente entre os homens, sendo em geral, desencadeado a partir da atração física, mas não necessariamente desprovido de sentimento 10,14,16. A estratégia do "ficar" várias vezes pode, inclusive, visar à efetivação de uma relação sexual, e posteriormente vir a se desdobrar em namoro 14 . Entretanto, essas diferenças não parecem interferir de modo substancial na utilização de métodos na primeira relação sexual de homens e mulheres.

As expectativas modernas de relações sexuais protegidas e a contradição relativa à norma social quanto ao modo de entrada na sexualidade parecem dar sustentação ao padrão observado: as moças, mais do que os rapazes, declararam ter sido motivadas pelo amor, com maior intervalo de tempo entre o início do relacionamento e a concretização do ato sexual, no contexto de uma relação estável (namoro, união ou casamento), ainda que parte delas tenha declarado sentiremse pressionadas pelos parceiros. Os rapazes sexualizaram rapidamente os relacionamentos, sendo estes na sua maioria ocasionais, em geral com amigas, motivados pelo "tesão", embora tenham também relatado medo, padrão este observado em vários estudos 7,16,17,18,19,20. É possível que a referência das mulheres ao amor possa ter sido influenciada pela alta proporção de entrevistadas que se mantiveram com o parceiro da iniciação sexual.

A diferença entre os sexos é consistente com diversos estudos: homens iniciam a vida sexual mais cedo do que as mulheres, e podem ter menos dificuldades para relatar este evento, ou até mesmo exagerar o relato, diminuindo a idade 3,15 . A explicação para tais diferenças tem ocorrido em parte pelas questões de gênero, no cumprimento de um determinado padrão de masculinidade ainda arraigado, implicando autocobrança, assim como na importância atribuída à virgindade entre as mulheres, que experimentam sua sexualidade mais freqüentemente em relacionamento estável do tipo "namoro". Há ainda, entre elas, a possibilidade de sub-relato de relações sexuais, aumento da idade em que estas se iniciam, ou declaração de sua ocorrência em relações estruturadas, em função das normas sociais 2,6,9,12,19,21.

É fato, por exemplo, que a pressão social para a iniciação sexual dos rapazes como prova de masculinidade aparece como um diferencial de gênero importante ao dissociar o sexo do amor. Nesse contexto, o início "tardio" da experimentação sexual aparece como motivo de preocupação 22. Para as mulheres, embora historicamente tenha se modificado a exigência da iniciação sexual somente após o casamento, a virgindade permanece uma referência importante em relações que entrelaçam afetividade e sexualidade 17,22,23. Tais concepções atuam como elementos que dificultam a interação entre os parceiros, bem como a negociação em torno do uso de métodos contraceptivos e de proteção ${ }^{17}$. Os homens enfatizam mais os aspectos que contribuem para a afirmação da identidade masculina 16,18,24. Ainda assim, isso varia a depender do tipo de relacionamento, seja o "ficar" - relação eventual e sem compromisso - ou o "namoro" - que implica laço afetivo, compromisso e fidelidade -, em decorrência da avaliação subjetiva dos riscos à saúde 16,18,24 .

Reconhece-se que em relações de gênero hierárquicas e desiguais, a vontade dos homens poderá prevalecer. Entretanto, a conversa prévia entre parceiros sobre gravidez e meios para evitar filhos, bem como a ocorrência da iniciação sexual em motéis, pressupõem intencionalidade, planejamento, negociação e possível interesse do casal pelas conseqüências sociais da sexualidade 10,14,25. O fato de acontecer "de repente" não quer dizer que esta não estava sendo esperada 16. É bem verdade que o nível de escolaridade, assim como as diferenças relacionadas ao intervalo de tempo entre o início do relacionamento e a primeira relação sexual, podem influenciar a conversa antecipada sobre a prevenção da gravidez 26 .

Entretanto, ainda que a simples menção à existência de conversa sobre prevenção de gravidez nada revele sobre o grau de concordância sobre o tema, os resultados sugerem mudanças importantes, também observadas em outros estudos, que precisam ser melhor investigadas 27,28 . Deve ser levado em consideração que as mulheres tendem a se iniciar sexualmente com homens mais velhos e experientes sexualmente 3 ,com maior poder para impor suas vontades, o que pode redobrar as desigualdades de gênero. É possível que a condição de maior autonomia desses homens influencie as decisões do casal de maneira diferenciada quanto ao uso de proteção, se comparada àquela em que ambos são adolescentes e inexperientes sexualmente, ou quando a parceira é mais velha

Mesmo assim, a preocupação do casal com o uso da contracepção reforça o argumento da mudança que vem sendo observada em relação ao envolvimento dos homens jovens nas questões de contracepção. Supostamente o uso do preservativo - o método mais prevalente entre mulheres e homens - requer a cooperação e a concordância de ambos os parceiros 27,28. Em contrapartida, a adoção do uso da pílula reafirma a contracepção como de responsabilidade feminina. Tal distinção sugere mudanças em relação à irresponsa- 
bilidade sexual e imaturidade psicológica atribuída aos jovens na iniciação sexual, bem como, no compartilhamento de responsabilidades, na medida em que a prática contraceptiva resulta de uma série de racionalidades complexas que se articulam entre si 27,28 , que não se constituem objeto de análise do presente estudo.

Para as mulheres, pertencer a famílias com renda mensal per capita acima de três salários mínimos, ser branca, ter obtido informações sobre gravidez e métodos contraceptivos em revistas femininas, ter conversado previamente com o parceiro sobre prevenção da gravidez, ter tido a primeira relação sexual em motel, ter 16 anos ou mais à época da iniciação sexual e ter parceiro paciente, tranqüilo, calmo e atencioso na ocasião, são condições que estiveram associadas ao uso de contraceptivos na primeira relação sexual. Entre os rapazes, esse se mostrou associado à sua ocorrência em relacionamento estável e em motel, à conversa prévia sobre prevenção de gravidez, além do fato de eles terem 15 ou mais anos de idade e a parceira ter se mostrado também paciente, tranqüila, calma e atenciosa.

A prevalência de contracepção na iniciação sexual revelou-se mais alta do que a observada na década de noventa, ratificando a tendência dos jovens se iniciarem em relações sexuais mais protegidas 4 . O aumento do uso do preservativo na iniciação sexual tem sido observado em outros estudos, com prevalência diferenciada quando o relacionamento é estável ou casual 3,15. Embora seja difícil precisar se tal conduta refere-se à prevenção da gravidez ou das DST/AIDS, ou de ambas, a mudança no padrão de métodos utilizados é corroborada pelo aumento na declaração do uso de preservativo 3,5 .

O recorte racial revelou uma maior proteção das mulheres brancas mesmo após o controle da renda e da escolaridade da mãe. Esse achado suscita novas investigações que possam aprofundar a compreensão sobre os aspectos socioculturais não contemplados neste estudo.

A constatação de que as revistas femininas como fonte de informações sobre meios para evitar gravidez está associada ao maior uso de contraceptivos merece mais atenção. A diversidade dos temas tratados, bem como a inclusão de matérias ou correspondências que retratam dúvidas e relatos de outras jovens em seus relacionamentos, pode preencher lacunas importantes relativas à falta de comunicação familiar e à inadequação dos conteúdos dos textos educativos que, em geral, enfatizam as dimensões científicas e técnicas e desconsideram os aspectos subjetivos e relacionais.

Apesar da importância atribuída à comunicação de mães e pais com suas filhas e filhos, pouco se sabe sobre a qualidade e a freqüência em que esta ocorre, e se permite a problematização dos seus conteúdos por parte das/os jovens, com base em suas próprias experiências. Além disso, muitas vezes, de modo explícito ou não, os pais estimulam a sexualidade masculina como afirmação da identidade e virilidade, e reprimem a feminina no intuito de postergar a primeira relação sexual das meninas, reforçando a importância da virgindade e do envolvimento emocional 22,29. A comunicação mais aberta entre adultos e jovens merece ser analisada para desvelar sua influência na comunicação entre parceiros por ocasião da iniciação sexual, ou no seu retardo, assim como, o impacto na adoção de práticas contraceptivas 10,28 .

Por fim, o aumento do uso de preservativo tem sido observado no Brasil ${ }^{3}$. Podemos supor que se a proteção na primeira relação sexual prediz comportamentos futuros, os resultados indicam a diminuição do risco de gravidez, o que deve ser mais bem investigado. Se ao contrário, a adoção de práticas contraceptivas na iniciação sexual não prediz comportamentos futuros, é necessário compreender melhor de que maneira o contexto dos relacionamentos afetivo-sexuais e as experiências reprodutivas, especialmente a ocorrência de gravidez e o aborto induzido, podem modificar o padrão observado na iniciação sexual.

A heterogeneidade sócio-cultural entre as cidades pesquisadas, com maior prevalência do uso de contraceptivos em Porto Alegre e menor em Salvador 10, demanda novas investigações, uma vez que na maior capital da Região Sul a iniciação sexual se dá mais precocemente, para ambos os sexos, mas de forma mais protegida do que na maior capital da Região Nordeste. Além disso, há uma homogeneidade no relato de proteção pelos homens das três cidades, em contraste com a maior diversidade entre as mulheres, sobretudo, quanto às variáveis de socialização e entrada na sexualidade, ao contexto dos relacionamentos e características das/os jovens e seus parceiro/as.

O presente estudo fornece evidências de que a idade à época da iniciação sexual permanece sendo um preditor importante para a utilização de métodos contraceptivos. Entretanto, a utilização em outros estudos de diferentes grupamentos etários, com adoção de pontos de corte equivalentes para homens e mulheres, dificulta a comparação. Assumir a idade da iniciação sexual como principal marcador do início da exposição ao risco é desconsiderar a tendência crescente de relações protegidas entre jovens e a necessidade de ampliar a discussão sobre suas trajetórias afetivo-sexuais. 
Há necessidade de novas investigações que possam ampliar o potencial explicativo das práticas contraceptivas na iniciação sexual, sendo fundamental incluir jovens de ambos os sexos, residentes em cidades de porte médio e na zona rural, para aprofundar o entendimento das mudanças ocorridas no comportamento dos/ as adolescentes. Assim, será possível desenhar propostas de intervenção efetivas no campo da saúde pública que considerem os direitos sexuais e reprodutivos de mulheres e homens jovens.

\section{Resumo}

Este estudo investigou o uso de contraceptivos na primeira relação sexual de 2.790 homens e mulheres. Trata-se de inquérito domiciliar em três capitais brasileiras, com entrevistas de amostra probabilística (Pesquisa GRAVAD). Utilizou-se análise de regressão logística. As variáveis foram agrupadas em: determinantes macrossociais, socialização e entrada na sexualidade, contexto da iniciação sexual e características da/o jovem e da/o parceira/o. A prevalência de foi de 68,3\% e de 65,3\% na dos homens. Entre elas, a contracepção associou-se à: renda familiar per capita, cor/raça e revistas femininas como fontes de informação sobre gravidez e contracepção. Para ambos os sexos, o uso foi mais freqüente quando houve conversa prévia sobre o tema entre parceiros, a iniciação sexual foi mais tardia e em motel, e o/a parceiro/a paciente. O tempo entre o início do relacionamento e a iniciação sexual mostrou-se associado ao uso na iniciação sexual dos rapazes. Fatores macrossociais parecem determinar a contracepção mais freqüente na iniciação sexual das mulheres, enquanto para os homens o contexto relacional é mais importante.

Comportamento Contraceptivo; Sexualidade; Identidade de Gênero

\section{Colaboradores}

L. F. B. Marinho colaborou na análise, interpretação dos dados e redação do artigo. E. M. L. Aquino participou da concepção e coordenação do estudo em todas as suas etapas, incluindo análise dos dados e redação do artigo. M. C. C. Almeida participou da construção e gerenciamento da base de dados, e contribuiu na análise de dados.

\section{Agradecimentos}

A Pesquisa GRAVAD - Gravidez na Adolescência: Estudo Multicêntrico sobre Jovens, Sexualidade e Reprodução no Brasil - foi elaborada por Maria Luiza Heilborn (Instituto de Medicina Social, Universidade do Estado do Rio de Janeiro), Michel Bozon (Institut National d'Études Démographiques, Paris), Estela M. L. Aquino (MUSA - Programa Integrado de Pesquisa e Cooperação Técnica em Gênero e Saúde, Universidade Federal da Bahia), Daniela Knauth (Núcleo de Pesquisa em Antropologia do Corpo e da Saúde, Universidade Federal do Rio Grande do Sul). Foi realizada com o apoio da Fundação Ford e seus principais resultados encontram-se publicados em O Aprendizado da Sexualidade: Reprodução e Trajetórias Sociais de Jovens Brasileiros (Rio de Janeiro: Editora Garamond; 2006). 


\section{Referências}

1. Bajos N, Ferrand M, Hassoun D. Au risque de léchec: la contraception au quotidien. In: Bajos N, Ferrand M; l'Équipe GINÊ, editors. De la contraception à l'avortement: sociologie des grossesses non prévues. Paris: Institut National de la Santé de la Recherche Médicale; 2002. p. 33-78.

2. Darroch JE, Singh S, Forst JJ. Differences in teenage pregnancy rates among five developed countries: the roles of sexual activity and contraceptive use. Fam Plann Perspect 2001; 33:244-50.

3. Paiva V, Calazans G, Venturi G, Dias R. Idade e uso de preservativo na iniciação sexual de adolescentes brasileiros. Rev Saúde Pública 2008; 42 Suppl 1:45-53.

4. Ferraz EA, Ferreira IQ, Morris L, Siu C, Negrão IP. Adolescentes, jovens e a Pesquisa Nacional Sobre Demografia e Saúde: um estudo sobre fecundidade, comportamento sexual e saúde reprodutiva. Rio de Janeiro: Bem-Estar Familiar no Brasil; 1999.

5. Centro Brasileiro de Análise e Planejamento. Comportamento sexual da população brasileira e percepções do HIV/AIDS. http://www.aids.gov.br/ final/biblioteca/avalia4/home.htm (acessado em 21/Out/2005).

6. Islas LAP, Allende MV. Entorno social, comportamiento sexual y reproductivo en la primera relación sexual de adolescentes estudiantes de escuelas públicas e privadas. In: Stern C, Echarri CJ, editors. Salud reproductiva y sociedad: resultados de investigación. México DF: El Colégio do México; 2000. p. 177-97.

7. Ferraz EA, Ferreira IQ, Soares MP, Morris L. Pesquisa sobre saúde reprodutiva e sexualidade do jovem 1989-1990: Rio de Janeiro, Curitiba e Recife. Rio de Janeiro: Bem-Estar Familiar no Brasil; 1992.

8. Rieth F. Amor e sexualidade. In: Béria J, organizador. Ficar, transar... a sexualidade do adolescente em tempos de AIDS. Porto Alegre: Tomo Editorial; 1998. p. 15-26.

9. Carvalho AA. Experiência sexual e anticoncepção. In: Bastos AVB, Morris L, Fernandez SR, organizadores. Saúde e educação sexual do jovem: um estudo em Salvador. Salvador: Universidade Federal da Bahia; 1989. p. 55-72.

10. Bozon M, Heilborn ML. Iniciação à sexualidade: modos de socialização, interações de gênero e trajetórias individuais. In: Heilborn ML, Aquino EML, Bozon M, Knauth DR, organizadores. O aprendizado da sexualidade: reprodução e trajetórias sociais de jovens brasileiros. Rio de Janeiro: Editora Garamond; 2006. p. 155-206.

11. Aquino EML, Araújo MJ, Almeida MC. Pesquisa GRAVAD: aspectos metodológicos, operacionais e éticos. In: Heilborn ML, Aquino EML, Bozon M, Knauth DR, organizadores. O aprendizado da sexualidade: reprodução e trajetórias sociais de jovens brasileiros. Rio de Janeiro: Editora Garamond; 2006. p. 88-139.

12. Leal AF. Uma antropologia da experiência amorosa: estudo de representações sociais sobre sexualidade [Dissertação de Mestrado]. Porto Alegre: Universidade Federal do Rio Grande do Sul; 2003.

13. Victora CG, Huttly SR, Fuchs SC, Olinto MT. The role of conceptual frameworks in epidemiological analysis: a hierarchical approach. Int J Epidemiol 1997; 26:224-7.
14. Aquino EML, Heilborn ML, Knauth DR, Bozon M, Almeida MCC, Araújo MJ, et al. Adolescência e reprodução no Brasil: a heterogeneidade dos perfis sociais. Cad Saúde Pública 2003; 19 Suppl 2: S377-88.

15. Rocha CLA, Horta BL, Pinheiro RT, Cruzeiro ALS, Cruz S. Use of contraceptive methods by sexually active teenagers in Pelotas, Rio Grande do Sul State, Brazil. Cad Saúde Pública 2007; 23:2862-8.

16. Almeida MCC, Aquino EML, Gaffkin J, Magnani RJ. Uso de contracepção por adolescentes de escolas públicas na Bahia. Rev Saúde Pública 2003; 37:566-75.

17. Alan Guttmacher Institute. In their own right: addressing the sexual and reproductive health needs of men worldwide. New York/Washington DC: Alan Guttmacher Institute; 2003.

18. Schuch P. "Ficar" ou namorar: eis a questão? Relações de gênero, afeto e corpo entre jovens universitários de Porto Alegre. Revista Brasileira de Sociologia da Emoção 2003; 1:312-36.

19. Lauritsen JI, Swicegood G. The consistency of selfreported initiation of sexual activity. Fam Plann Perspect 1997; 29:215-21.

20. Chirinos JL, Salazar VC, Brindis CD. A profile of sexually active male adolescent high school students in Lima, Peru. Cad Saúde Pública 2000; 16:733-46.

21. Necchi S, Schuffer MR. Adolescente varón: iniciación sexual y anticoncepción (Argentina). Rev Chil Pediatr 2001; 72:159-68.

22. Castro MG, Abramovay M, Silva LB. Juventudes e sexualidade. Brasília: Organização das Nações Unidas para a Educação, a Ciência e a Cultura; 2004.

23. Laumann EO, Gagnon JH, Michaels S. The social organizations of sexuality: sexual practices in the United States. Chicago: University of Chicago Press; 1994.

24. Bajos N, Durand S. Teenage sexual and reproductive behavior in developed countries: country report for France. New York/Washington DC: Alan Guttmacher Institute; 2001. (Occasional Report, 5).

25. Rieth F. A iniciação sexual na juventude de mulheres e homens. Horizontes Antropológicos 2002; 8:77-91.

26. Gage AJ. Sexual activity and contraceptive use: the components of the decision making process. Stud Fam Plann 1998; 29:154-66.

27. Manning WD, Longmore MA, Giordano PC. The relationship context of contraceptive use at first intercourse. Fam Plan Perspect 2000; 32:104-10.

28. Stone N, Ingham R. Factors affecting British teenagers contraceptive use at first intercourse: the importance of partner communication. Perspect Sex Reprod Health 2002; 34:191-7.

29. Stern C, Medina G. Adolescencia y salud en México. In: Oliveira MC, organizador. Cultura, adolescência e saúde: Argentina, Brasil, México. Campinas: Consórcio de Programas em Saúde Reprodutiva e Sexualidade na América Latina; 2000. p. 44-97.

Recebido em 01/Set/2008

Versão final reapresentada em 29/Jan/2009

Aprovado em 06/Abr/2009 